\title{
Psychoendokrinologie: Trends und ihr Bezug zur Praxis
}

\author{
Ulrike Ehlert \\ Klinische Psychologie und Psychotherapie, Universität Zürich, Schweiz
}

\section{Schlüsselwörter \\ Psychoendokrinologie - Diagnostik . \\ Sexualhormone $\cdot$ Cortisol}

\section{Zusammenfassung}

Die Psychoendokrinologie ist ein interdisziplinäres Forschungs- und Arbeitsgebiet, das mit experimentellen Untersuchungen an Gesunden und Patienten die Bedeutung hormoneller Systeme auf das biopsychosoziale Gesamtgefüge im Kontext von Gesundheit und Krankheit aufklärt. Neben dem autonomen Nervensystem und der Hypothalamus-Hypophysen-Nebennieren-Achse als den etablierten Stresssystemen werden in der aktuellen Forschung vermehrt geschlechtsspezifische und altersbezogene Einflussfaktoren auf die psychobiologische Verarbeitung von akuten, chronischen und/oder traumatischen Belastungen einbezogen. Für die psychotherapeutische Praxis sind psychoendokrinologische Forschungsergebnisse aus 3 Gründen von Bedeutung: Verbesserung der Differenzialdiagnostik, Entwicklung individueller Krankheitsmodelle und Therapieevaluation.

\section{Einleitung}

Das Forschungs- und Arbeitsfeld der Psychoendokrinologie ist ein junges und sich ständig erweiterndes interdisziplinäres Forschungsfeld, an dem vor allem die Neurowissenschaften, die Medizin und die Psychologie beteiligt sind. Die Grundfragestellung bezieht sich auf die Zusammenhänge zwischen Hormonen und Verhalten. In experimentellen Untersuchungen zeigte sich bereits früh, dass es keinen einfachen bidirektionalen Weg zwischen Hormonen und Verhalten gibt, sondern dass ein komplexes Modell von Interaktionen zwischen Umwelt, physiologischer Verarbeitung dieser Umweltreize,

\author{
Keywords \\ Psychoendocrinology - Diagnostic procedures . \\ Sex hormones · Cortisol
}

\section{Summary \\ Psychoendocrinology: Trends and Their Relation to \\ Practice}

Psychoendocrinology is an interdisciplinary field of research in healthy subjects and patients, addressing the relevance of hormonal systems in the overall context of biopsychosocial explanations for health and disease, by experimental approaches. The autonomous nervous system and the hypothalamus-pituitary-adrenal axis are well-known hormonal stress systems contributing to the physiological effects of acute, chronic, and/or traumatic stress. Recent research has broadened the view on ageand sex-related modulators of stress reactions in diverse human populations. The psychoendocrine findings have 3-fold clinical relevance: increase of diagnostic knowledge on patient subgroups, development of individual illness concepts, and evaluation of psychotherapeutic interventions.

endokrinen und immunologischen Reaktionen und daraus resultierenden Verhaltensreaktionen notwendig ist. Die Komplexität dieser Prozesse erfordert reduktionistische Forschungsstrategien, die erst bei gleichzeitiger Berücksichtigung verschiedener Studienresultate ein etwas klareres Bild zu ausgewählten Aspekten der Körper-Psyche-Interaktion erlauben.

Grundsätzlich wird ein seriöser wissenschaftlicher Zugang zu der oben genannten Komplexität dadurch erleichtert, dass bestimmte Forschungsparadigmen und etablierte Messmethoden für die Untersuchung der jeweiligen Fragestellungen herangezogen werden. Deshalb werden im Folgenden das Stresskonzept als Beispiel eines zentralen Forschungsparadigmas

\section{KARGER \\ Fax +497614520714 \\ Information@Karger.com}

www.karger.com
(C) 2014 S. Karger GmbH, Freibur

$1016-6262 / 14 / 0242-0125 \$ 39.50 / 0$

Accessible online at:

www.karger.com/ver
Prof. Dr. Ulrike Ehlert

Klinische Psychologie und Psychotherapie

Universität Zürich

Binzmühlestrasse 14/26, 8050 Zürich, Schweiz

u.ehlert@psychologie.uzh.ch 
der Psychoendokrinologie und verschiedene Hormonachsen mit ihren jeweils sezernierten Hormonen als Wirk- oder Messgrößen aufgezeigt. Auf dieser Grundlage aufbauend werden ausgewählte Trends der aktuellen Psychoendokrinologie und ihr Bezug zur praktischen Arbeit vorgestellt.

\section{Stress}

Das Wort Stress ist fester Bestandteil des allgemeinen Sprachgebrauchs, wobei es notwendig ist, zwischen Stressor und Stressreaktion zu differenzieren. Aufbauend auf die Definition von Selye [1956], der zufolge Stress die unspezifische körperliche Anstrengung als Antwort auf Anforderungen der Umwelt beschreibt, ist es heute nachgewiesen, dass in Abhängigkeit von der Stress auslösenden Situation (Stressor), die von einer Alltagsanforderung bis zu einem Trauma reichen kann, eine Vielzahl unterschiedlicher emotionaler, kognitiver, physiologischer und verhaltensbezogener Reaktionen beobachtbar ist. Der Interaktion zwischen Umweltanforderungen und individueller Reaktivität wurde von Lazarus und Folkman [1984] durch die Beschreibung kognitiver Bewertungsprozesse (Bewertung nach dem Bedrohungsausmaß und der zur Verfügung stehenden Ressourcen) Rechnung getragen. Die Unterscheidung zwischen Auslöser (Stress) und Reaktion, auch als Coping bezeichnet, sowie die Komplexität des Verarbeitungsprozesses auf der psychischen und physiologischen Ebene sind in Abbildung 1 zusammengefasst.

Die Verarbeitung von Stress und die daraus resultierende Anpassung an diese Gegebenheiten wird durch eine Vielzahl von Faktoren wie genetische Merkmale, frühere Erfahrungen, Entwicklungsprozesse, psychologische Merkmale sowie körperliche Voraussetzungen beeinflusst. In Abhängigkeit vom individuellen Zusammenwirken dieser Faktoren und dem Ausmaß der Stressoren kommt es zu Resilienz und damit zur erfolgreichen Anpassung an Lebensumstände oder zu Fehlanpassungen wie beispielsweise dem Burnout-Syndrom. Die Fehlanpassungen können dann im ungünstigsten Fall zu manifesten Krankheiten und Störungen wie Angststörungen, Depression, Suchtmittelabhängigkeit, Herz-Kreislauf- oder Autoimmunerkrankungen führen [vgl. Ehlert et al., 2013]. An diesem Anpassungsgeschehen sind auf zentralnervöser und peripherer Ebene unterschiedliche endokrine Organe und die von ihnen sezernierten Hormone beteiligt.

\section{Gut erforschte Hormonsysteme und ihr Zusammenhang mit Stress}

Eine direkte Konsequenz von akutem und/oder chronischem Stress ist die Freisetzung verschiedener Hormone wie Cortisol, Adrenalin und Noradrenalin, die einerseits infolge einer Aktivierung des autonomen Nervensystems ansteigen und andererseits aufgrund der Aktivierung der Hypothala-

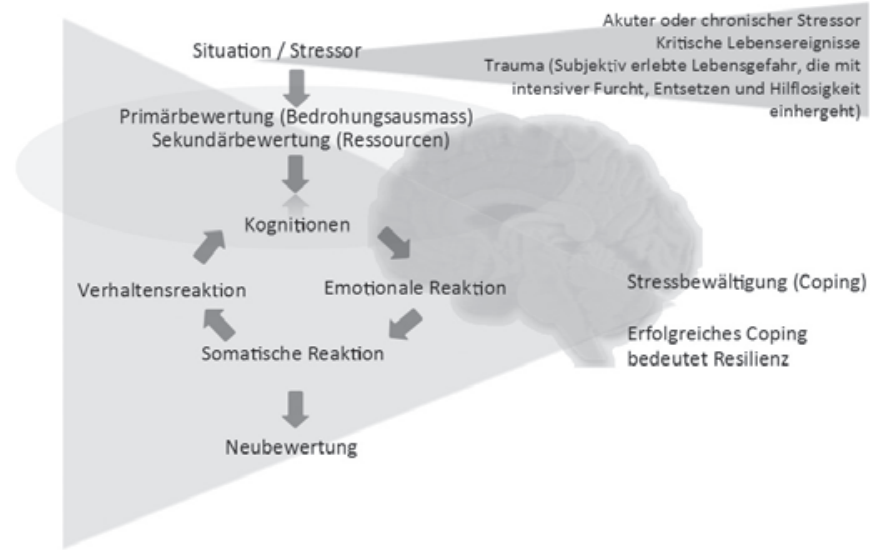

Abb. 1. Unterscheidung zwischen Stressor und Stressreaktion.

mus-Hypophysen-Nebennieren-Achse (HHNA) freigesetzt werden. Diese stressbedingten Hormonfreisetzungen, ihre Rezeptoren in verschiedensten zellulären Strukturen, ihre Feedbackwirkung auf die jeweilige Hormonachse und ihre Modulation durch Enzyme oder weitere Hormone stellen ein Kernforschungsfeld der Psychoendokrinologie dar.

In den letzten 20 Jahren konzentrierte sich die einschlägige Forschung auf die beiden oben genannten Stresssysteme [vgl. Bremner et al., 1996; Habib et al, 2001] und ihre Bedeutung für depressive Störungen und Angststörungen sowie psychosomatische Erkrankungen. Bestimmte Formen depressiver Störungen, insbesondere die unipolare Depression, sind mit einer Überfunktion der HHNA assoziiert, wohingegen bei somatoformen Störungen und der Posttraumatischen Belastungsstörung eine Hypofunktion vorliegt [vgl. Heim et al., 2000; Ehlert et al., 2001]. Für die von hypocortisolären Patienten häufig geschilderten Schmerzsymptome stellt der Hypocortisolismus insofern eine physiologische Erklärung dar, als eine reduzierte Cortisolfreisetzung mit einer verstärkten Synthese von Prostaglandinen (Schmerzmediatoren) einhergeht. Die Cortisolhyporeaktivität zeigt sich bei Patientinnen mit chronischen Unterbauchbeschwerden ohne Organkorrelat, bei einem Teil der Patienten und Patientinnen mit Reizdarmsyndrom, bei Whiplash-Syndrom oder orofazialen Schmerzen. Bei allen genannten Schmerzgruppen lassen sich keine eindeutigen organischen Schmerzkorrelate, jedoch überdurchschnittlich häufig traumatische Erfahrungen und/oder chronischer Stress nachweisen [Ehlert et al., 1999, 2005; Gaab et al., 2005a; Galli et al., 2009].

Die Erforschung des Enzyms Alpha-Amylase, das unter anderem im Speichel sezerniert wird, stellt für psychoendokrinologische Fragestellungen einen interessanten Parameter dar, da die Alpha-Amylase als indirekter Indikator der durch Stress bedingten noradrenergen Freisetzung angesehen werden kann [vgl. Nater und Rohleder, 2009; Nater et al., 2007]. Unter körperlichem, pharmakologischem wie auch psychosozialem Stress kommt es zu einem nachweisbaren Anstieg der Konzentration dieses Enzyms. Die Messung des Alpha-Amy- 
lase-Spiegels erübrigt bei bestimmten Fragestellungen dadurch die Venipunktion für Blutabnahmen zur Bestimmung der Katecholaminspiegel von Adrenalin und Nordarenalin [Nater et al., 2005; Ehlert et al., 2006].

\section{Die Dissoziation von physiologischer und psychischer Stressantwort}

In experimentellen psychoendokrinologischen Untersuchungen an Gesunden und an Patientenpopulationen bezieht sich eine zentrale Fragestellung auf die Korrelation zwischen der subjektiv berichteten und der physiologischen Stressantwort. Mason [1968] zufolge wirkt sich psychosozialer Stress insbesondere dann steigernd auf die HHNA aus, wenn der Stressor für die betroffene Person bedeutsam, neuartig, unvorhersagbar und unkontrollierbar ist. Der Trier Social Stress Test (TSST) [Kirschbaum et al., 1993] erfüllt in hohem Maße diese Anforderungen, da mit dieser standardisierten Stressprovokation mentale, emotionale und physiologische Stressreaktionen mit großer Zuverlässigkeit ausgelöst werden können [vgl. Dickerson und Kemeny, 2002]. Sowohl Gaab et al. [2005b] als auch Schlotz et al. [2011] konnten mit unterschiedlichen statistischen Herangehensweisen aufzeigen, dass die selbstberichteten kognitiven Bewertungsprozesse (Bedrohungsausmaß und Ressourcen zur Bewältigung) in signifikantem Zusammenhang mit der Gesamtcortisolfreisetzung vor, während und nach der Stressprovokation mittels TSST standen. Je belastender der Stresstest antizipiert wurde, desto höher war die Freisetzung von Cortisol. In einer umfassenden Zusammenschau zur emotionalen Reaktivität (Prä-postVergleich) auf den TSST fand sich ein entsprechender Zusammenhang mit physiologischen Parametern (mehrheitlich Cortisolfreisetzung), jedoch nur bei einem Viertel aller methodisch vergleichbaren Studien [Campbell und Ehlert, 2012]. Entsprechend der aktuellen Befundlage ist davon auszugehen, dass eine Reihe von moderierenden Variablen wie Persönlichkeitsmerkmale, motivationale Faktoren und soziale Erwünschtheit die nur teilweise zu beobachtende Konvergenz von emotionaler und physiologischer Stressreaktion beeinflussen.

\section{Befunde zu geschlechtsspezifischen Unterschieden}

In den letzten Jahren ist innerhalb der Psychoendokrinologie ein Trend zu komplexeren Untersuchungsdesigns zu beobachten, bei denen beispielsweise in Stressprovokationsstudien gezielt Moderatoren der Stressantwort als unabhängige Variable in den Studienplan einbezogen werden. So wird beispielsweise der unterschiedlichen Stressreaktivität von Männern und Frauen Rechnung getragen und es werden unterschiedliche Lebensphasen berücksichtigt, wie z.B. bei Frauen das gebärfähige Alter im Vergleich zur Postmenopausenzeit.

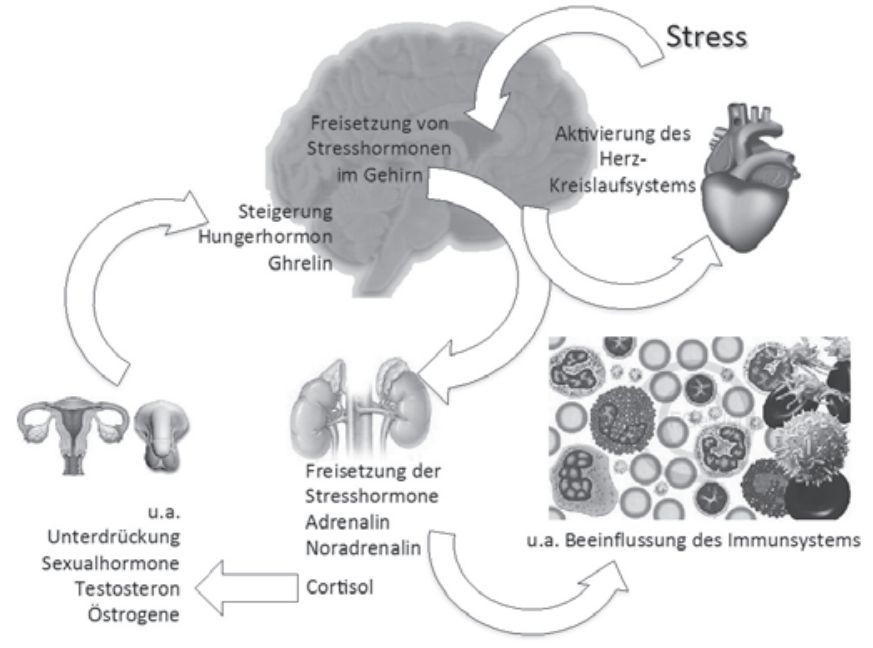

Abb. 2. Hormonelle Konsequenzen von Stress.

In verschiedenen Studien hat sich gezeigt, dass Frauen im gebärfähigen Alter unter standardisierten Stressbedingungen (TSST) einen geringeren Cortisolanstieg aufweisen als Männer [vgl. Hammerfald et al., 2006]. Da diese supprimierte Cortisolantwort jedoch bei postmenopausalen Frauen nicht zu beobachten ist [Lindheim et al., 1994], scheint der Effekt durch Sexualsteroide vermittelt zu werden. So konnten Lindheim und Kollegen zeigen, dass eine transdermale Applikation von Östrogen einen Cortisol hemmenden Effekt besitzt. Umgekehrt ist bekannt, dass eine Aktivierung der HHNA und der daraus resultierenden erhöhten Cortisolfreisetzung zu einer Suppression der Hypothalamus-Hypophysen-Gonaden-Achse führt, die sowohl bei Männern als auch bei Frauen mit einer reduzierten Freisetzung von Sexualhormonen (Testosteron und Östrogene) assoziiert ist [Handa und Weiser, 2013]. Eine reduzierte Fertilität kann demzufolge als ein Resultat erhöhter Cortisolspiegel, z.B. infolge von chronischem Stress, angesehen werden [vgl. Berga und Naftolin, 2012].

Bezüglich der Sexualhormonfreisetzung findet sich ein weiterer geschlechtsspezifischer Effekt für Frauen mit einem intakten Menstruationszyklus: Kurz vor der Ovulation ist die Freisetzung von Östrogen im Vergleich zu allen anderen Zykluszeitpunkten am höchsten. Wird nun die Kalorienaufnahme pro Tag während des gesamten Zyklus untersucht, zeigt sich, dass zum Ovulationszeitpunkt die Kalorienaufnahme bis zu $200 \mathrm{kcal}$ pro Tag niedriger ist als im restlichen Zyklus und dass dieser Effekt durch die erhöhten Östrogenspiegel zu erklären ist, da diese einen dämpfenden Einfluss auf das «Hunger»-Hormon Ghrelin nehmen [Asarian und Geary, 2006]. Vergleichbare Variationen der Kalorienaufnahme lassen sich bei Männern nicht nachweisen.

Eine Zusammenschau der aufgezeigten psychoendokrinologischen Vorgänge als Folge von Stress findet sich in Abbildung 2. 


\section{Konsequenzen für die Praxis}

Wenngleich psychoendokrinologische Grundlagenforschung auf den ersten Blick keinen direkten «Nutzen» für die praktische psychotherapeutische Arbeit mit Patientinnen und Patienten hat, sollte dieser Eindruck aus 3 Gründen relativiert werden. Die Ergebnisse psychoendokrinologischer Grundlagenstudien dienen erstens einer verbesserten Differenzialdiagnostik, zweitens unterstützen sie die Akzeptanz psychobiologischer Krankheitsmodelle und können drittens dazu herangezogen werden, die Evaluation psychotherapeutischer Interventionen zu objektivieren.

Die differenzialdiagnostische Charakterisierung von Patientensubpopulationen anhand endokriner Parameter bedeutet nicht nur eine diagnostische Absicherung, sondern auch die Möglichkeit einer präziseren Indikationsstellung für psychotherapeutische Maßnahmen, da beispielsweise Schmerzsymptomatiken ohne Organkorrelat physiologisch durch die Erhebung unstimulierter Cortisolspiegel objektiviert werden können.

Viele Patientinnen und Patienten mit funktionellen Beschwerden leiden darunter, dass somatomedizinische Untersuchungen keinen Hinweis auf eine körperliche Ursache ihrer Beschwerden geben. Wird ihnen in der Folge unauffälliger medizinischer Untersuchungsresultate eine psychotherapeuti- sche Behandlung empfohlen, fühlen sie sich häufig unverstanden und lehnen diesen Vorschlag ab. Werden jedoch psychoendokrinologische Befunde in die Erklärung ihrer Symptomatik einbezogen, wird die Wahrscheinlichkeit des Verständnisses dafür vergrößert, dass Stressoren und/oder Traumata zu physiologischen (Fehl-)Anpassungen führen können, die in der jeweiligen, vermeintlich funktionellen Symptomatik resultieren. Die Erarbeitung eines individuellen Krankheitsmodells, unter Einbeziehung der psychophysiologischen Fehlanpassungen, kann den Zugang zu einer stressabhängigen Sichtweise der Symptomatik erleichtern und die Akzeptanz psychotherapeutischer Interventionen ermöglichen.

Die Wirksamkeit kognitiv-verhaltenstherapeutischer Interventionen im Sinne einer Beschwerdereduktion und einer Wiederherstellung psychobiologischer Funktionsfähigkeit lässt sich durch die wiederholte Messung hormoneller Parameter dokumentieren [vgl. beispielsweise Michopoulos et al., 2013] und dürfte die Akzeptanz psychotherapeutischer Interventionen auch außerhalb der klassisch psychopathologischen Arbeitsfelder deutlich erhöhen.

\section{Disclosure Statement}

Die Autorin erklärt hiermit, dass keinerlei Interessenskonflikte in Bezug auf dieses Manuskript bestehen.

\section{Literatur}

Asarian L, Geary N: Modulation of appetite by gonadal steroid hormones. Philos Trans R Soc Lond B Biol Sci 2006;361:1251-1263.

Berga S, Naftolin F: Neuroendocrine control of ovulation. Gynecol Endocrinol 2012;28(suppl 1):9-13.

Bremner JD, Krystal JH, Southwick SM, Charney DS: Noradrenergic mechanisms in stress and anxiety: II. Clinical studies. Synapse 1996;23:39-51.

Campbell J, Ehlert U: Acute psychosocial stress: does the emotional stress response correspond with physiological responses? Psychoneuroendocrinology 2012;37:1111-1134

Dickerson SS, Kemeny ME: Acute stressors and cortisol reactivity: a meta-analytic review. Psychosomatics 2002;54:105-123.

Dhlert U, Heim C, Hellhammer DH: Chronic pelvic pain as a somatoform disorder. Psychother Psychosom 1999;68:87-94.

Ehlert U, Gaab J, Heinrichs M: Psychoneuroendocrinological contributions to the etiology of depression, posttraumatic stress disorder, and stress-related bodily disorders: the role of the hypothalamus-pituitary-adrenal axis. Biol Psychol 2001:57:141-152.

Ehlert U, Nater UM, Böhmelt A: High and low unstimulated salivary cortisol levels correspond to different symptoms of functional gastrointestinal disorders. J Psychosom Res 2005;59:7-10.

Ehlert U, Erni K, Hebisch G, Nater U: Salivary alphaamylase levels after yohimbine challenge in healthy men. J Clin Endocrinol Metab 2006;91:5130-5133.

Ehlert U, La Marca R, Abbruzzese EA, Kübler U: Biopsychologie. Stuttgart, Kohlhammer, 2013.
Gaab J, Baumann S, Budnoik A, Gmünder H, Hottinger N, Ehlert U: Reduced reactivity and enhanced negative feedback sensitivity of the hypothalamuspituitary-adrenal axis in chronic whiplash-associated disorder. Pain 2005a;119:219-224.

Gaab J, Rohleder N, Nater UM, Ehlert U: Psychological determinants of the cortisol stress response: the role of anticipatory cognitive appraisal. Psychoneuroendocrinology 2005b;30:599-610.

Galli U, Gaab J, Ettlin DA, Ruggia F, Ehlert U, Palla S: Enhanced negative feedback sensitivity of the hypothalamus-pituitary-adrenal axis in chronic myogenous facial pain. Eur J Pain 2009;13:600-605.

Habib KE, Gold PW, Chrousos GP: Neuroendocrinology of stress. Endocrinol Metab Clin North Am 2001;30:695-728.

Hammerfald K, Eberle C, Grau M, Kinsperger A, Zimmermann A, Ehlert U, Gaab J: Persistent effects of cognitive-behavioral stress management on cortisol responses to acute stress in healthy subjects - a randomized controlled trial. Psychoneuroendocrinology 2006;31:333-339.

Handa RJ, Weiser MJ: Gonadal steroid hormones and the hypothalamo-pituitary-adrenal axis. Front Neuroendocrinol 2014;35:197-220.

-Heim C, Ehlert U, Hellhammer DH: The potential role of hypocortisolism in the pathophysiology of stress-related bodily disorders. Psychoneuroendocrinology 2000;25:1-35.

Kirschbaum C, Pirke KM, Hellhammer DH: The 'Trier Social Stress Test' - a tool for investigating psychobiological stress responses in a laboratory setting. Neuropsychobiology 1993;28:76-81.

Lazarus RS, Folkman S: Stress, Appraisal and Coping. New York, Springer, 1984.
Lindheim SR, Legro RS, Morris RS, Wong IL, Tran DQ, Vijod MA, Stanczyk FZ, Lobo RA: The effect of progestins on behavioral stress responses in postmenopausal women. J Soc Gynecol Investig 1994;1:79-83.

Mason JW: A review of psychoendocrine research on the sympathetic-adrenal medullary system. Psychosomatics 1968;30(suppl 5):631-653.

Michopoulos V, Mancini F, Loucks TL, Berga SL: Neuroendocrine recovery initiated by cognitive behavioral therapy in women with functional hypothalamic amenorrhea: a randomized, controlled trial. Fertil Steril 2013;99:2084-2091.

Nater UM, Rohleder N: Salivary alpha-amylase as a non-invasive biomarker for the sympathetic nervous system: current state of research. Psychoneuroendocrinology 2009;34:486-496.

Nater UM, Rohleder N, Gaab J, Berger S, Jud A, Kirschbaum C, Ehlert U: Human salivary alphaamylase reactivity in a psychosocial stress paradigm. Int J Psychophysiol 2005;55:333-342.

Nater UM, Rohleder N, Schlotz W, Ehlert U, Kirschbaum C: Determinants of the diurnal course of salivary alpha-amylase. Psychoneuroendocrinology 2007;32:392-401.

Schlotz W, Hammerfald K, Ehlert U, Gaab J: Individual differences in the cortisol response to stress in young healthy men: testing the roles of perceived stress reactivity and threat appraisal using multiphase latent growth curve modeling. Biol Psychol 2011;87:257-264.

Selye H: The Stress of Life. New York, McGraw-Hill, 1956. 